\title{
Using Chaos Eye Method to Diagnose Power Capacitors
}

\author{
Meng-Hui Wang ${ }^{1, *}$ \\ Guang-Ci Ye ${ }^{1}$ \\ Meng-Ju Hsieh ${ }^{1}$ \\ Kuo-Nan $\mathrm{Yu}^{1}$ \\ Shiue-Der $\mathrm{Lu}^{2}$ \\ No. 35, Lane 215, Sec.1, Chung-Shan Rd., Taiping District, Taichung City 411, Taiwan. \\ ${ }^{2}$ Chung Yuan Christian University, Department of Electrical Engineering \\ No. 200 Chung Pei Road, Chung Li District, Taoyuan City 320, Taiwan. \\ *Corresponding Author's E-mail address: wangmh@ncut.edu.tw
}

\begin{abstract}
This study focused on the measurement and diagnostic analysis of partial discharge in power capacitors operating under prolonged periods of high voltage. An off-line test was conducted on two power capacitors, which were subjected to defect preprocessing before the experiment. The partial discharge test of the terminal to the outer casing was carried out using a $24 \mathrm{kV}$ high voltage transformer. The voltage of the power discharge, after which the voltage signals of the test voltage and the partial discharge were extracted. The characteristics of the discharge signal were identified using an oscilloscope. The chaos synchronization detection method was then employed to establish a chaotic error scatter plot for the discharge voltage he gravity (chaos eye) of the error scatter plot was used as the fault diagnosis feature. The advantage of this approach was the drastic reduction of the amount of data for feature extraction, and the effective detection of the slight discharge signal variation in power capacitors, thereby anticipating their operation and enabling the implementation of preemptive contingency measures to avoid major accidents.
\end{abstract}

Keywords: chaos synchronization detection method; fault diagnosis; partial discharge; power capacitators

\section{INTRODUCTION}

Quality of life and economic development often depend on the safe and stable operation of power systems. However, prolonged periods of high temperature, voltage, and loading lead to the aging or fail of electrical equipment. Resent, power system maintenance is carried out by the Taiwan Power Company through periodic maintenance checks. However, a substantial increase in transformer substations and equipment, as well as the exceedingly long period between maintenance checks, the safe operation of electrical equipment. If electrical equipment failure anticipated, replaced, the operating costs of equipment downtime be drastically lowered, thereby reducing economic losses. Therefore, the realtime monitoring of power capacitors.

According to recent research, common power capacitor faults include damping, insulation aging, support frame discharge, insulating oil spills, connection strap exfoliation, and immersion failures [1-3]. This study explored the fault types by subjecting the power capacitors to defect damage and subsequently increasing the voltage using a highvoltage transformer to test their insulation systems [4].
A defective or aged insulation system can result in penetration damage the internal insulation of the power capacitor by discharge pulses ext, the partial discharge signal of the power capacitor terminal to the ground was measured using a sensor [5], and the fault feature was analyzed through chaos synchronization.

The chaos synchronization detection method is a product of chaos theory, which was proposed by meteorologist Edward Norton Lorenz to explore the unstable conditions of nonlinear dynamic systems. The primary characteristic of this instability is that even a slight change in the default initial conditions within the chaotic system can eventually lead to considerably varying outcomes after a long period of amplification effects [6]. Therefore, this study employed the chaos synchronization detection method to generate chaos eye coordinates by using the discharge signals measured from power capacitors, and used these coordinates as the fault diagnosis feature.

\section{Partial Discharge Test of Power CAPACITOR}

This study mainly measured the partial discharge signal (a pulse signal) that occurred during an off-line test. Because the extracted discharge signal cycle was long and had a high frequency, a considerable amount of data were involved.

Excessive interference during the test the partial discharge signal. Therefore, to extract the high frequency signal from the partial discharge signal, the lower frequency signal and noises were filtered out using a high-pass filter [7].

\section{A. Off-line Test}

The off-line test involves the disconnection of the power supply with the main goal of testing the insulation performance of the power capacitor, which in this study on AC withstand voltage. The voltage of the single phase to ground terminal of the power capacitor was increased using a $24 \mathrm{kV}$ transformer, a high frequency current transformer (HFCT) was connected to the ground terminal [8]. The signal was received using an oscilloscope and transmitted to a computer through a data acquisition system (DAQ), as shown in Fig. 1. When the insulation of the singlephase capacitor group reached a critical voltage point, the discharge impulse gradually penetrated the insulation layer and resulted in damage. At this point, 
the conductive characteristics enabled the pulse to flow to the ground terminal, leading to the coupling of the discharge pulse to the HFCT sensor.

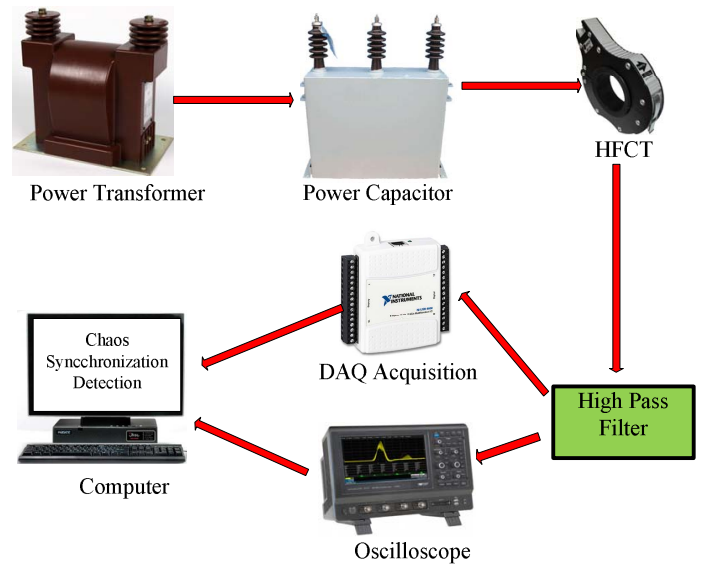

Figure. 1. Power capacitor measurement system

\section{B. Defect Construction in Power Capacitors}

Constructing defects in the power capacitors was difficult because of their simple structure. The two power capacitors used in this study (Fig. 2) were deprecated products provided by local industries, in which the oxidation of insulating oil led to the expansion of the shell [9]. Fig. 3 shows the capacitor constructed as wear damage the terminal metal casing [10].

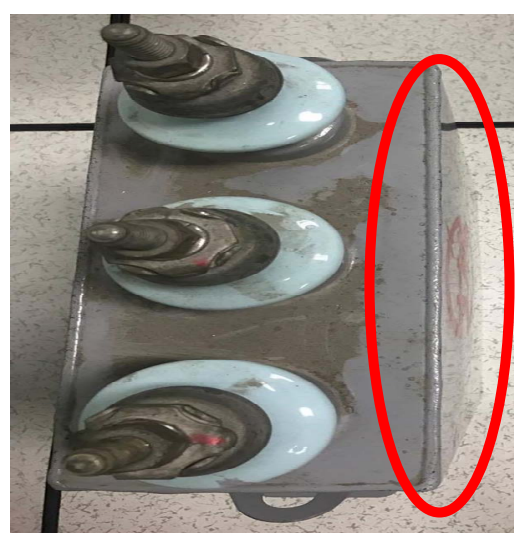

Figure. 2. Expansion of power capacitor shell

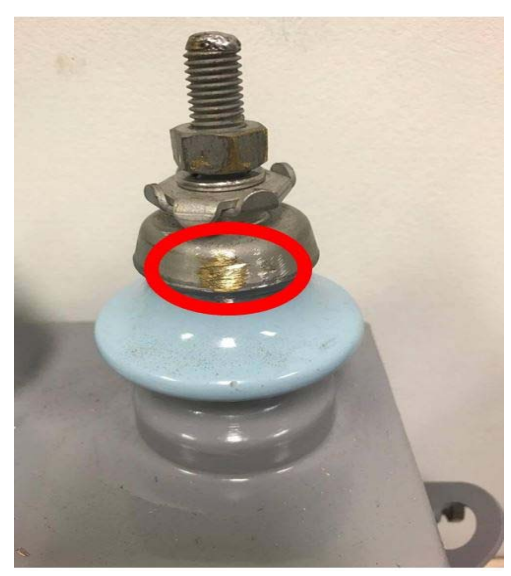

Figure. 3. Wear damage power capacitor metal casing

\section{High-Pass Filter Design}

Because of noise interference during the actual measurement, a high-pass filter was added to increase the accuracy of the measured results. In addition, for the extracted discharge signal to have a longer shock effect and become more easily identifiable, the highpass filter was produced using a passive filter [11]. The cut-off frequency $f_{c}(6 \mathrm{MHz})$ and the frequency response are shown in Figs. 4 and 5, respectively.

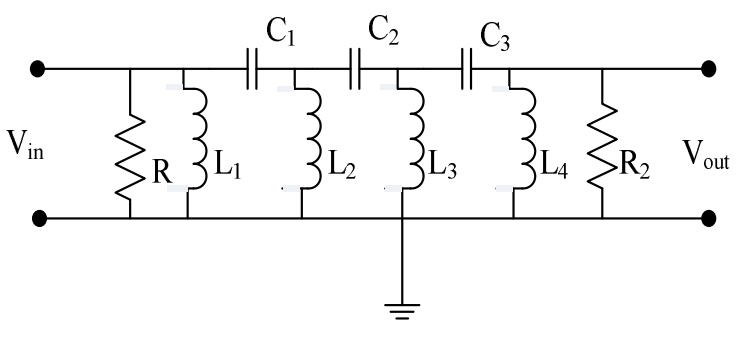

Figure. 4. Circuit diagram of high-pass filter

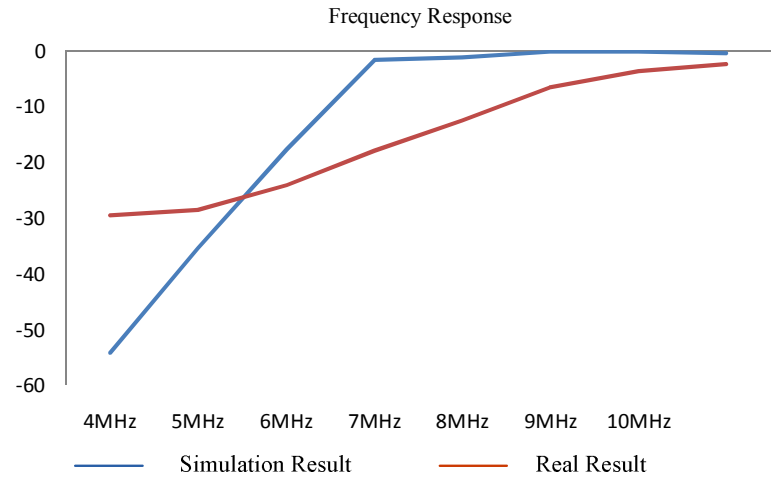

Figure. 5. Frequency response of high-pass filter

The actual test results for the high-pass filter indicate that the output signal was zero at $34 \mathrm{MHz}$, with only a small fraction of the $6 \mathrm{MHz}$ signal passing through (Fig. 5). The signal passed through when the frequency was between 9 and $10 \mathrm{MHz}$, mainly because of the presence of impedance in the circuit itself and the cable during the actual circuit measurement. $\omega_{L}$ was minimal at low frequency has no effect, but maximal at high frequency, causing a -3 $\mathrm{dB}$ time attenuation in the circuit.

\section{FEATURES EXTRACTION}

The two chaotic systems in a chaos synchronization system can be called the master and slave systems. Synchronization results from using the slave system to track the signals of the master system. In addition, varying default values cause different degrees of chaos in the trajectories of both systems. The characteristic values obtained in this study were the dynamic errors of the master and slave systems coupled under normal conditions. Thus, a subtraction of chaos in both systems can be used to identify dynamic error variations. The master $\left(S_{\text {master }}\right)$ and slave $\left(S_{\text {slave }}\right)$ of a master/slave system are expressed in (1) and (2), respectively. 


$$
\begin{gathered}
S_{\text {master }}=\left\{\begin{array}{l}
\dot{x_{1}}=f_{1}\left(x_{1}, x_{2}, \ldots, x_{n}\right) \\
\dot{x_{2}}=f_{2}\left(x_{1}, x_{2}, \ldots, x_{n}\right) \\
\vdots \\
\dot{x_{n}}=f_{n}\left(x_{1}, x_{2}, \ldots, x_{n}\right)
\end{array}\right. \\
S_{\text {slave }}=\left\{\begin{array}{l}
\dot{y_{1}}=f_{1}\left(x_{1}, x_{2}, \ldots, x_{n}\right) \\
\dot{y_{2}}=f_{2}\left(x_{1}, x_{2}, \ldots, x_{n}\right) \\
\vdots \\
\dot{y}_{n}=f_{n}\left(x_{1}, x_{2}, \ldots, x_{n}\right)
\end{array}\right.
\end{gathered}
$$

Where $f_{i}(=1,2, \ldots, n)$ is a nonlinear function. By subtracting (1) and (2), the master and slave dynamic error is generated, as expressed in (3). A calculation yielded the chaotic dynamic function as. (4).

$$
\begin{aligned}
& \left\{\begin{array}{l}
e_{1}=y_{1}-x_{1} \\
e_{2}=y_{2}-x_{2} \\
\vdots \\
e_{n}=y_{n}-x_{n}
\end{array}\right. \\
& \left\{\begin{array}{l}
\dot{e_{1}}=f_{1}\left(x_{1}, x_{2}, \ldots, x_{n}\right)-f_{1}\left(y_{1}, y_{2}, \ldots, y_{n}\right) \\
\dot{e_{2}}=f_{2}\left(x_{1}, x_{2}, \ldots, x_{n}\right)-f_{2}\left(y_{1}, y_{2}, \ldots, y_{n}\right) \\
\vdots \\
\dot{e_{1}}=f_{n}\left(x_{1}, x_{2}, \ldots, x_{n}\right)-f_{n}\left(y_{1}, y_{2}, \ldots, y_{n}\right)
\end{array}\right.
\end{aligned}
$$

The Lorenz Chaos System is employed for this study. The master $\left(L_{\text {master }}\right)$ and slave $\left(L_{\text {slave }}\right)$ Lorenz systems are expressed in (5) and (6), respectively.

$$
\begin{gathered}
L_{\text {master }}=\left\{\begin{array}{l}
\dot{x_{1}}=\alpha\left(x_{2}-x_{1}\right) \\
\dot{x_{2}}=\beta x_{1}-x_{1} x_{3}-x_{2} \\
\dot{x_{3}}=x_{1} x_{2}-\psi_{3}
\end{array}\right. \\
L_{\text {slave }}=\left\{\begin{array}{l}
\dot{y_{1}}=\alpha\left(y_{2}-y_{1}\right) \\
\dot{y_{2}}=\beta y_{1}-y_{1} y_{3}-y_{2} \\
\dot{y}_{3}=y_{1} y_{2}-y_{3}
\end{array}\right.
\end{gathered}
$$

By subtracting (5) and (6), the chaotic dynamic function of Lorenz master/slave system is obtained in the form of matrix shown as (7).

$$
\left[\begin{array}{c}
\dot{e}_{1} \\
\dot{e_{2}} \\
\dot{e_{3}}
\end{array}\right]=\left[\begin{array}{ccc}
-\alpha & \alpha & 0 \\
\beta & -1 & 0 \\
0 & 0 & -\gamma
\end{array}\right]\left[\begin{array}{l}
e_{1} \\
e_{2} \\
e_{3}
\end{array}\right]+\left[\begin{array}{c}
y_{2} y_{3}-x_{2} x_{3} \\
-y_{1} y_{3}+x_{1} x_{3} \\
y_{1} y_{2}-x_{1} x_{2}
\end{array}\right]
$$

$\mathrm{X}$ is the master system with an initial value of zero; $\mathrm{y}$ is the slave system and contains original signal values; and the adjusted error coefficients are set as $\alpha=10, \beta=28$ and $\gamma=-(8 / 3)$, respectively and are used to generate the dynamic map of chaotic dynamic error. The coordinates of the two centers of gravity in the map are defined as the chaos eyes, which are used as the features of the power capacitors [12].

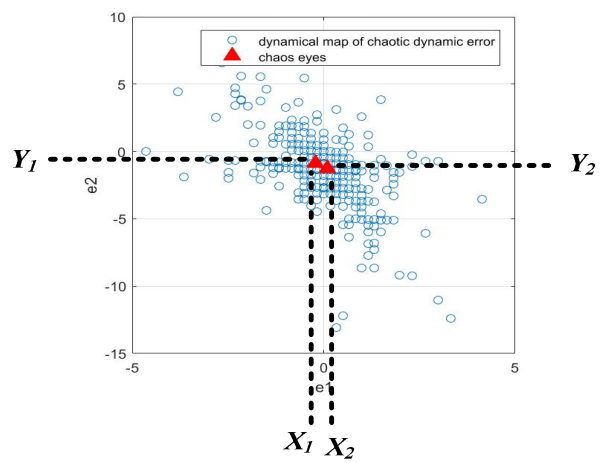

Figure. 6. Chaos eye scatter plot

\section{Actual Measurement AND Results ANALYSIS}

Fig. 7 shows the actual measurement wiring, whereby the voltage of the power capacitor was continuously increased by the voltage transformer until the occurrence of partial discharge. The HFCT, used as the sensor for extracting the partial discharge signal, was connected to the ground terminal of the power capacitor. The extracted signal changes were transmitted to the filter circuit through a Bayonet Neill-Concelman (BNC) connector, the partial discharge signal of the power capacitor was analyzed by computer. The main system within the chaos synchronization test detection system was set to zero and viewed as the benchmark for the power capacitor under normal conditions, its scatter plot was saved in the database for chaos synchronization detection.

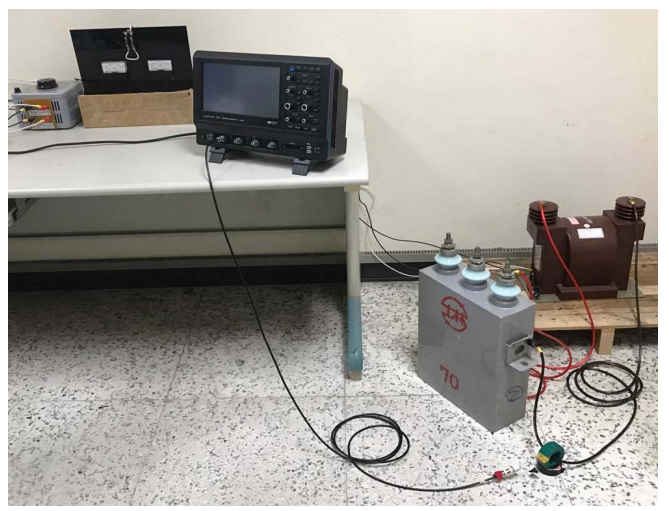

Figure. 7. Actual measurement wiring

Fig. 8 shows the partial discharge response characteristics of the power capacitors in the off-line test under the two fault features insufficient insulating oil and worn metal casing. The yellow line represents the mains voltage signal and the red line represents the partial discharge signal of the power capacitor ground terminal. Fig. 8(a) demonstrated more frequent discharge under the condition of insufficient insulating oil; Fig. 8(b) shows a low discharge, though with a larger voltage amplitude. The gravity of the fault 
features were obtained through chaos synchronization detection (Fig. 9). The chaos eye coordinates are shown in Table 1.

The amount of data generated from the discharge signal of the power capacitor surpassed 1 million units and the discharge waveform did not reveal clear characteristics. Therefore, the chaos synchronization detection method was employed to process the original discharge signal data, and the coordinates of the centers of gravity in the dynamical map were used as the characteristic values for identifying data features. The advantage of this method was its ability to effectively compress a substantial amount of data and derive clear characteristic values.

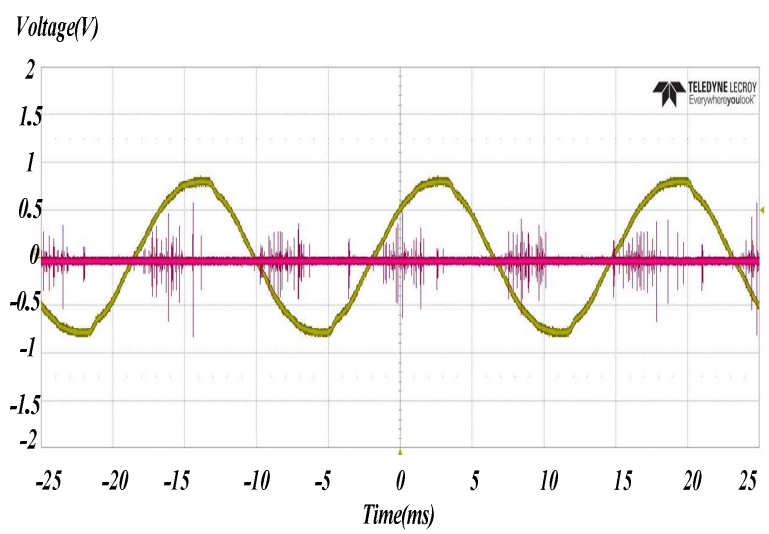

(a)

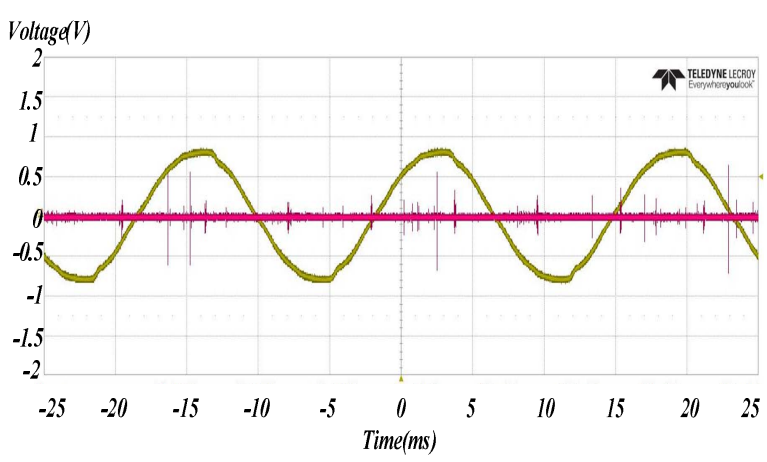

(b)

Figure. 8. Discharge waveform of power capacitator with (a) insufficient insulating oil (b) worn metal casing

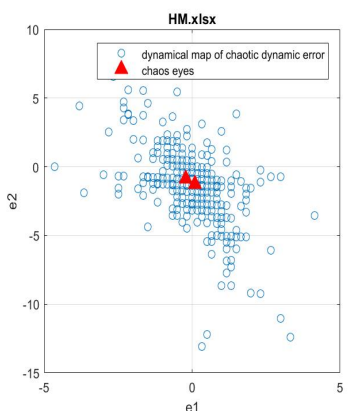

(a)

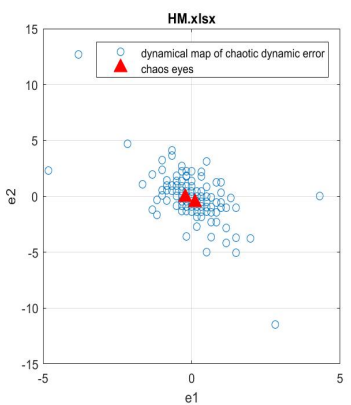

(b)
Figure. 9. Chaos scatter plots and gravity for power capacitors with (a) insufficient insulating oil (b) worn metal casing
Table 1. Chaos eye coordinates for each power capacitor fault

\begin{tabular}{|c|c|c|}
\hline Chaos Eye & $\begin{array}{c}\text { Fault category } \\
\text { insufficient } \\
\text { insulating oil }\end{array}$ & $\begin{array}{c}\text { worn metal } \\
\text { casing }\end{array}$ \\
\hline $\mathbf{X 1}$ & -0.2044 & -0.2186 \\
\hline $\mathbf{X} 2$ & 0.0883 & 0.1134 \\
\hline Y1 & -0.8580 & -0.0693 \\
\hline Y2 & -1.2739 & -0.5721 \\
\hline
\end{tabular}

Fig. 9(a) shows the dynamic error distribution points and gravity of the power capacitors under the condition of insufficient insulating oil. The dynamic error distribution points in the figure were mostly concentrated between 5 and -5 , with the highest value being 6 and the lowest value being approximately -13 . The dynamic error distribution point changes in Fig. 9(b) were obvious and mostly concentrated between 3 and -3 . The distribution points and gravity of the chaotic characteristic waveform were obtained after the chaotic dynamic error scatter plots of the two fault features (Fig. 9). Power capacitor fault identifications and classification, according to the chaotic characteristic waveform distributions and gravity with greater differences.

\section{CONCLUSION}

The partial discharge phenomenon currently major approach for fault diagnosis. This study proposed using the voltage from the partial discharge as the fault detection signal power capacitors, extracting the slight variation in the discharge voltage parameter using chaos synchronization detection. The gravity in the chaos scatter diagrams can serve as the basis for power capacitor fault detection in the future. The following conclusions are proposed based on the research results:

1). The chaos synchronization detection method proposed in this study can be incorporated into other methods such as extension theory, to diagnose the types and categories of power capacitor faults.

2). The chaos synchronization detection method employed in this study operates rapidly in a simple program and achieves high accuracy in detecting power capacitor faults.

3). This study proposed using the voltage of the partial discharge as the signal diagnose power capacitors, previous detection methods. However, the severity of partial discharge cannot be judged from a single phenomenon; rather it should be determined through months or years of long-term monitoring. The goal of online real-time monitoring can be achieved in the future through integration. 


\section{ACKNOWLEDGEMENT}

The authors are grateful to the Ministry of Science and Technology for funding this research (Project No: 105-2221-E-167-014-MY3).

\section{REFERENCES}

[1] J. Li, S. Jiao, Y. Wen and H. Wang, "Online Insulation Monitoring System of High-voltage Capacitive Substation Equipment Based on WSN," CICED Proceedings, pp. 1-6, 2010

[2] C. Feng, J. Daozhuo, F. Yu and G. Hongjie, "Research on Power Capacitor Internal Fault Criterion," Fifth International Conference on Intelligent Systems Design and Engineering Applications, pp. 841-844, 2014.

[3] B. Liu, W. Chen, W. Shang, Q. Liu, L. Chu and S. Chen, "The Influence of the Partial Discharge on the Electrical Aging Property of High Voltage Ceramic Capacitor," International Symposium on Electrical Insulating Materials, pp. 763-765, 1998.

[4] L. Cheng, Z. Li and W. Liu, "Design Optimization of Power Capacitor Major Insulation Based on Partial Discharge Performance," International Conference on Condition Monitoring and Diagnosis (CMD), pp. 282-286, 2016.

[5] K. M. Burke, W. J. Sarjeant, J. L. Zirnheld, L. Bragg, D. Perry, K. Slenes, et al., "Partial Discharge Characteristics of Low Kilovolt Class Capacitors," IEEE Pulsed Power Conference, pp. 974-977, 2005.
[6] C. H. Huang, C. H. Lin and C. L. Kuo, "Chaos Synchronization-Based Detector for Power-quality Disturbances Classification in a Power System, " IEEE Transactions on Power Delivery, Vol. 26, Issue. 2, pp. 944953, Dec. 2010

[7] X. Q. Dang, J. Y. Liu, N. Liu, X. Lei and K. Yang, "Research of On-line Detection for Power Capacitor," International Conference on Electronic Packaging Technology, pp. 1-4, 2005.

[8] X. Gu, Y. Xu, R. Xia, S. Meng and Y. Wang, "On-line Calibration of Partial Discharge Monitoring for Power Cable by HFCT Method," IEEE Electrical Insulation Conference (EIC), pp. 40-44, 2016.

[9] G. R. Newcomb, "Film/Foil Power Capacitor," IEEE International Conference on Electrical Insulation, pp. 72-72, 1980

[10] F. C. Gu, H. C. Chang and C. C. Kuo, "Gas-Insulated Switchgear PD Signal Analysis Based on Hilbert-huang Transform with Fractal Parameters Enhancement," IEEE Transactions on Dielectrics and Electrical Insulation, Vol. 20, pp. 1049-1055, 2013.

[11] P. Bidan, T. Lebey and C. Neacsu, "Development of a New Off-line Test Procedure for Low Voltage Rotating Machines Fed by Adjustable Speed Drives (ASD)," IEEE Transactions on Dielectrics and Electrical Insulation, Vol. 10, pp. 168175, 2003.

[12] H. T. Yau and M. H. Wang, "Chaotic Eye-Based Fault Forecasting Method for Wind Power Systems, " IET Renewable Power Generation, Vol. 9, Issue. 6, pp. 593-599, Oct. 2015. 